Mots. Les langages du politique

$97 \mid 2011$

Les collectivités territoriales en quête d'identité

\title{
Actualité bibliographique Les langages du politique
}

Josette Lefèvre

\section{(2) OpenEdition}

Journals

Édition électronique

URL : https://journals.openedition.org/mots/20557

DOI : $10.4000 /$ mots. 20557

ISBN : 15/11/2013

ISSN : 1960-6001

Éditeur

ENS Éditions

Édition imprimée

Date de publication : 15 novembre 2011

ISBN : 978-2-84788-326-8

ISSN : 0243-6450

Référence électronique

Josette Lefèvre, «Actualité bibliographique Les langages du politique », Mots. Les langages du politique [En ligne], 97 | 2011, mis en ligne le 08 mars 2012, consulté le 23 avril 2022. URL : http:// journals.openedition.org/mots/20557 ; DOI : https://doi.org/10.4000/mots.20557

(c) ENS Éditions 


\section{Actualité bibliographique Les langages du politique}

A Contrario, 2011, n¹6, L'argumentation au carrefour des disciplines. Sciences du langage et sciences sociales, R. Micheli éd., Lausanne, BSN Press, $180 \mathrm{p}$.

Banda Daniel, Moure José éd., 2011, Le cinéma. L'art d'une civilisation, 1920-1960, Paris, Flammarion, $487 \mathrm{p}$.

BAnoun Bernard, EnderLe-RISTORI Michaela, LE MoËLSylvie éd., 2011, Migrations, exil et traduction, Tours, Presses universitaires François Rabelais, $430 \mathrm{p}$.

Belletante Joseph, 2011, Séries et politique. Quand la fiction contribue à l'opinion, Paris, L'Harmattan, $268 \mathrm{p}$.

BESNARD Tiphaine, 2010, Les prostituées à la Salpêtrière et dans le discours médical, 1850-1914. Une folle débauche, Paris, L'Harmattan, $224 \mathrm{p}$.

BouRdon Jérôme, 2011, Du service public à la télé-réalité. Une histoire culturelle des télévisions européennes, 1950-2010, Paris, INA, 247 p.

Bourg EOIS Guillaume, YÈCHE Hélène éd., 2011, Signes, couleurs et images de l'Europe, Rennes, Presses universitaires de Rennes, $292 \mathrm{p}$.

BURger Marcel, Jacquin Jérôme, Michelı Raphaël éd., 2011, La parole politique en confrontation dans les médias, Louvain-la-Neuve, De Boeck.

CHANIAL Philippe, 2011, La sociologie comme philosophie politique et réciproquement, Paris, La Découverte, $300 \mathrm{p}$.

ChARLE Christophe, VINCENT Julien éd., 2011, La société civile. Savoirs, enjeux et acteurs en France et en Grande-Bretagne, 1780-1914, Rennes, Presses universitaires de Rennes, $320 \mathrm{p}$.

Chateauraynaud Francis, 2011, Argumenter dans un champ de forces. Essai balistique sociologique, Paris, Pétra, $484 \mathrm{p}$.

CHUQUeT Jean éd., 2011, Le langage et ses niveaux d'analyse. Cognition, production de formes, production du sens, préface de M. Audiffren, Rennes, Presses universitaires de Rennes, $196 \mathrm{p}$.

Communication \& Langages, 2011, n 168 , Les «petites phrases » en politique, A. KriegPlanque, C. Ollivier-Yaniv éd., Paris, Necplus, $139 \mathrm{p}$.

Corcuff Philippe, 2011, Les nouvelles sociologies. Entre le collectif et l'individuel, $3^{\mathrm{e}}$ édition actualisée, Paris, Armand Colin, $127 \mathrm{p}$.

Coulangeon Philippe, 2011, Les métamorphoses de la distinction. Inégalités culturelles dans la France d'aujourd'hui, Paris, Grasset, 165 p.

DelPhy Christine éd., 2011, Un troussage de domestique, Paris, Syllepse, 184 p. 
Delporte Christian, Maréchal Denis, Moine Caroline, Veyrat-Masson Isabelle éd., 2011, Images et sons de mai 68 (1968-2008), Paris, Nouveau Monde, 420 p.

Desalmand Paul, Stalloni Yves, 2011, 365 expressions expliquées, Paris, Chêne, 288 p.

Develotte Christine, Kern Richard, LAmy Marie-Noëlle éd., 2011, Décrire la conversation en ligne. Le face à face distanciel, Lyon, ENS Éditions, $224 \mathrm{p}$.

DuchêNE Alexandre, MoïsE Claudine éd., 2011, Langage, genre et sexualité, Paris, Nota Bene, $296 \mathrm{p}$.

GANDON Francis, 2011, La morale du linguiste. Saussure entre Affaire Dreyfus et massacre des Arméniens (1894-1898), Limoges, Lambert-Lucas, 96 p.

GIRARDOT Dominique, 2011, La société du mérite. Idéologie méritocratique et violence néolibérale, préface de A. Caillé et P. Chanial, Lormond, Le Bord de l'eau, 250 p.

Greffet Fabienne éd., 2011, Continuerlalutte.com. Les partis politiques sur le web, Paris, Presses de Sciences Po, $313 \mathrm{p}$.

HeRnANDEZ Severiano Rojo, 2011, Une guerre de papier. La presse basque antifasciste dans les années trente, Rennes, Presses universitaires de Rennes, $300 \mathrm{p}$.

HERVOUET-FARRAR Isabelle éd., 2011, Enfance et errance dans la littérature européenne du xIxe siècle, Clermont-Ferrand, Presses universitaires Blaise Pascal, 306 p.

LaLAnne Michèle, Cochoy Franck éd., 2010, Montrer le marché. Afficher, emballer, étiqueter, Toulouse, Presses universitaires du Mirail (Sciences de la société), 216 p.

Langage et société, 2011, n 137, Les discours de l'accompagnement. Nouvelles normes de retour à l'emploi, Paris, MSH, $158 \mathrm{p}$.

LAPLANTINE Chloé, 2011, édition, présentation et transcription, Baudelaire par Émile Benveniste. 370 pages manuscrites inédites (BNF et Collège de France), Limoges, Lambert-Lucas, $770 \mathrm{p}$.

LASSERre François, Garrigue Roland, 2011, Comme vache qui pisse et autres expressions animales, Lonay, Delachaux et Niestlé, $127 \mathrm{p}$.

Laval Christian, Vergne Francis, Clément Pierre, Dreux Guy, 2011, La nouvelle école capitaliste, Paris, La Découverte, $240 \mathrm{p}$.

LAZZARATo Maurizio, 2011, La fabrique de l'homme endetté. Essai sur la condition néolibérale, Paris, Éditions Amsterdam, $125 \mathrm{p}$.

LEBARON Frédéric, 2011, Les indicateurs sociaux au xxı siècle, Paris, Dunod, $120 \mathrm{p}$.

Lefort Geneviève, 2011, Olympe Gevin-Cassal (1849-1945). L'éducation des mères, Rennes, Presses universitaires de Rennes, $290 \mathrm{p}$.

LE Goff Jacques éd., 2011, Penser la crise avec Emmanuel Mounier, préface de J. Delors, Rennes, Presses universitaires de Rennes, 216 p.

Les DÉsobéISSANTS, 2011, Désobéir à l'argent, Le Pré-Saint-Gervais, Le Passager clandestin, $61 \mathrm{p}$.

MACHEREY Pierre, 2011, La parole universitaire. De Kant et Hegel à Lacan et Bourdieu, Paris, La Fabrique, $352 \mathrm{p}$.

MARICHEZ Jean, 2011, Croyances meurtrières. Essai pour la paix, Paris, L'Harmattan, 252 p. MARTIN Jean-Philippe, 2011, La confédération paysanne aujourd'hui. Un syndicat face aux défis duxxıe siècle, Paris, L'Harmattan, $214 \mathrm{p}$.

Michaud Yves, 2011, Qu'est-ce que le mérite?, Paris, La Découverte, 340 p.

Perreault Michel, 2011, Je ne suis pas une entreprise!, Paris, La Découverte, 224 p. 
Postel Nicolas, Cazal Didier, Chavy Frédéric, Sobel Richard éd., 2011, La responsabilité sociale de l'entreprise. Nouvelle régulation du capitalisme?, Villeneuve d'Ascq, Presses universitaires du Septentrion, $416 \mathrm{p}$.

RAFANell I ORRA Josep, 2011, En finir avec le capitalisme thérapeutique. Soin, politique et communauté, Paris, La Découverte, 350 p.

Roupnel-Fuentes Manuela, 2011, Les chômeurs de Moulinex, Paris, PUF, 359 p.

SePulchre Sarah éd., 2011, Décoder les séries télévisées, Louvain-la-Neuve, De Boeck, $256 \mathrm{p}$.

SOULET Marc-Henry éd., 2011, Ces gens-là. Les sciences sociales face au peuple, Fribourg, Academic Press Fribourg, 205 p.

STROH Wilfried, 2010, La puissance du discours, Paris, Les Belles Lettres, $514 \mathrm{p}$.

Texto! Textes \& Cultures, 2011, vol. XVI, nº 2, Céline Poudat éd., en ligne [http://www. revue-texto.net].

Toulemon Laurent, Mesté France, Véron Jacques éd., 2011, Dictionnaire démographique et des sciences de la population, Paris, Armand Colin, $528 \mathrm{p}$.

TRAIMOND Bernard, 2011, L'économie n'existe pas, Lormond, Le Bord de l'eau, 114 p.

Transform, hors série, 2011, Une crise de civilisation?, Colloque Espaces Marx 28-29 janvier 2011, Paris, Espaces Marx, 204 p.

Van Zanten Agnès, Rayou Patrick éd., 2011, Les 100 mots de l'éducation, Paris, PUF (Que sais-je?), $128 \mathrm{p}$.

VÉRINE Bertrand, DÉTRIE Catherine éd., 2011, L'actualisation de l'intersubjectivité. De la langue au discours, hommage à Jeanne-Marie Barbéris, Limoges, Lambert-Lucas, $190 \mathrm{p}$.

Von Münchow Patricia, 2011, Lorsque l'enfant paraît... Le discours des guides parentaux en France et en Allemagne, Toulouse, Presses universitaires du Mirail, 168 p.

Josette Lefèvre

Université de Picardie Jules Verne, CNRS (CURAPP)

josette.lefevre@u-picardie.fr 